\title{
Extended Poisson Fréchet Distribution and its Applications
}

Mohamed G. Khalil

Department of Statistics, Mathematics and Insurance,

Benha University, Benha, Egypt.

Email: hndaoy@gmail.com

Rezk H.

Mathematical Sciences Department, College of Science,

Princess Nourah bint Abdulrahman University, Riyadh, Saudi Arabia.

Department of Statistics, Al-Azhar University, Cairo, Egypt.

Email: hrmohamed@pnu.edu.sa

\begin{abstract}
A new version of the Fréchet model is investigated and studied. Some of its properties are mathematically derived. The well-known maximum likelihood classical method is used in estimating the unknown parameters. The new version is much better than other important competitive Fréchet versions in modeling reliability real data.
\end{abstract}

Keywords: Zero Truncated Poisson Distribution; Fréchet Distribution; Maximum Likelihood

\section{Introduction and physical motivation}

A random variable $(\mathrm{R}-\mathrm{V}) U$ is said to have the Fréchet (Fr) model if its probability density function (P-D-F) and survival function (S-F) are given by (for $u \geq 0$ )

and

$$
g_{F r}^{(\alpha, \beta)}(u)=u^{-(\beta+1)} \alpha^{\beta} \beta \exp \left[-u^{-\beta} \alpha^{\beta}\right]
$$

$$
1-G_{F r}^{(\alpha, \beta)}(u)=-\exp \left[-u^{-\beta} \alpha^{\beta}\right]+1,
$$

respectively, where $\alpha, \beta>0$. On the other hand, the probability mass function (P-M-F) of $T$ where $T$ has a zero truncated Poisson (Z-T-P) model is given as

$$
\left.P M F_{Z . T . P .}^{(\lambda)}(T=t)\right|_{(t=1,2, \ldots)}=\frac{\exp (-\lambda) \lambda^{t}}{t ! \mathrm{C}_{(\lambda)}}
$$

Where

$$
\mathrm{C}_{(\lambda)}=-\exp (-\lambda)+1
$$

Consider the Burr-X-Fr version defined by the cumulative distribution function (C-D-F) and P-D-F given by

and

$$
\mathbf{H}_{B X F r}^{(\theta, \alpha, \beta)}(z)=\left[-\exp \left(-\left\{\frac{\exp \left[-z^{-\beta} \alpha^{\beta}\right]}{1-\exp \left[-z^{-\beta} \alpha^{\beta}\right]}\right\}^{2}\right)+1\right]^{\theta} .
$$

$$
\mathbf{h}_{B X F r}^{(\theta, \alpha, \beta)}(z)=2 \theta \beta \alpha^{\beta} z^{-(\beta+1)}\left\{1-\exp \left[-z^{-\beta} \alpha^{\beta}\right]\right\}^{-3}
$$


respectively. Let

$$
\begin{gathered}
\times \exp \left[-2 z^{-\beta} \alpha^{\beta}\right] \exp \left(-\left\{\frac{\exp \left[-z^{-\beta} \alpha^{\beta}\right]}{1-\exp \left[-z^{-\beta} \alpha^{\beta}\right]}\right\}^{2}\right) \\
\times\left[-\exp \left(-\left\{\frac{\exp \left[-z^{-\beta} \alpha^{\beta}\right]}{1-\exp \left[-z^{-\beta} \alpha^{\beta}\right]}\right\}^{2}\right)+1\right]^{\theta-1},
\end{gathered}
$$

Then, the conditional C-D-F of $X \mid T$ is

$$
\min \left\{Y_{1}, Y_{2}, \cdots, Y_{T}\right\}=Z \text {. }
$$

$$
F(z \mid T)=1-\operatorname{Pr}(Z>t \mid T)=1-\left[1-\mathbf{H}_{B X F r}^{(\theta, \alpha, \beta)}(z)\right]^{T} .
$$

Therefore, the unconditional C-D-F of the Poisson Burr-X-Fr (P-BX-Fr) version, can be expressed as

$$
\left.F_{P B X F r}^{(\lambda, \theta, \alpha, \beta)}(z)\right|_{(\lambda \in R)}=\frac{1-\exp \left\{-\lambda\left[-\exp \left(-\left\{\frac{\exp \left[-z^{-\beta} \alpha^{\beta}\right]}{1-\exp \left[-z^{-\beta} \alpha^{\beta}\right]}\right\}^{2}\right)+1\right]^{\theta}\right\}}{\mathrm{C}_{(\lambda)}},
$$

and its corresponding P-D-F is

$$
\begin{gathered}
\left.f_{P B r X F r}^{(\lambda, \theta, \alpha, \beta)}(z)\right|_{(\lambda \in R)}=\frac{2 \theta \lambda \beta \alpha^{\beta}}{\mathrm{C}_{(\lambda)}} z^{-(\beta+1)}\left\{1-\exp \left[-z^{-\beta} \alpha^{\beta}\right]\right\}^{-3} \\
\times \exp \left(-2 z^{-\beta} \alpha^{\beta}-\left\{\frac{\exp \left[-z^{-\beta} \alpha^{\beta}\right]}{1-\exp \left[-z^{-\beta} \alpha^{\beta}\right]}\right\}^{2}\right) \\
\times\left[1-\exp \left(-\left\{\frac{\exp \left[-z^{-\beta} \alpha^{\beta}\right]}{\left.1-\exp \left[-z^{-1} \alpha\right)^{\beta}\right]}\right\}^{2}\right)\right]^{\theta-1} \\
\times \exp \left\{-\lambda\left[-\exp \left(-\left\{\frac{\exp \left[-z^{-\beta} \alpha^{\beta}\right]}{1-\exp \left[-z^{-\beta} \alpha^{\beta}\right]}\right\}^{2}\right)+1\right]^{\theta}\right\} .
\end{gathered}
$$

The hazard (failure) rate function (H-R-F) of the P-BX-Fr model can be calculated via $f_{P B r X F r}^{(\lambda, \theta, \alpha, \beta)}(z) /\left[1-F_{P B X F r}^{(\lambda, \theta, \alpha, \beta)}(z)\right]$. The P-D-F of the new Fr version can be right skewed and unimodal (see Figure 1) also it can be left skewed (see Table 1). The H-R-F of the new model can be bathtub (U), unimodal-bathtub (unimodal-U), increasing and decreasing. All Tables and Figures are listed in the Appendix. Some important Fr models are developed by Nadarajah and Kotz(2003), Barreto-Souza, et al. (2011), Krishna, et.al., (2013), Mead, et al., (2014), Yousof, et el., (2015), Mahmod and Mandoh (2013), Afify, et al., (2016-a and 2016-b), Yousof, et al., (2016), Korkmaz, et al., (2017), Yousof et al., (2018-a and 2018-b), Brito, et al., (2017), Hamedani, et al., (2017), Cordeiro, et al., (2018), Hamedani, et al., (2018), Korkmaz, et al., (2018), Chakraborty, et al., (2018), Korkmaz, et al., (2019), Hamedani, et al., (2019), among others.

\section{Mathematical properties \\ 2.1 Useful expansions}

Using

$$
\exp (\boldsymbol{\varphi})=\sum_{c=0}^{\infty} \varphi^{c} \frac{1}{c !}
$$


the P-D-F in (6) can be written as

$$
\begin{aligned}
& f_{P B X F r}^{(\lambda, \theta, \alpha, \beta)}(z)=\sum_{h=0}^{\infty} 2 \theta \lambda^{1+h} \beta \alpha^{\beta} \frac{(-1)^{h}}{h ! \mathrm{C}_{(\lambda)}} z^{-(\beta+1)} \\
& \times \exp \left[-\left(\frac{\exp \left[-z^{-\beta} \alpha^{\beta}\right]}{1-\exp \left[-z^{-\beta} \alpha^{\beta}\right]}\right)^{2}\right] \\
& \times \frac{\exp \left[-2 z^{-\beta} \alpha^{\beta}\right]}{\left\{1-\exp \left[-z^{-\beta} \alpha^{\beta}\right]\right\}^{3}}\left\{1-\exp \left[-\left(\frac{\exp \left[-z^{-\beta} \alpha^{\beta}\right]}{1-\exp \left[-z^{-\beta} \alpha^{\beta}\right]}\right)^{2}\right]\right\}^{\theta(h+1)-1} .
\end{aligned}
$$

If $|\varphi|<1$ and $p>0$ where $p$ is any real-non-integer, then

$$
(1-\boldsymbol{\varphi})^{\mathrm{p}}=\sum_{d=0}^{\infty} \frac{(-1)^{d} \Gamma(1+\mathrm{p})}{d ! \Gamma(1+\mathrm{p}-d)} \boldsymbol{\varphi}^{d}
$$

Applying (8) to (7) we get

$$
\begin{gathered}
f_{P B X F r}^{(\lambda, \theta, \alpha, \beta)}(z)=2 \theta \beta \alpha^{\beta} \frac{z^{-(\beta+1)} \exp \left[-2 z^{-\beta} \alpha^{\beta}\right]}{\mathrm{C}_{(\lambda)}} \\
\times \sum_{h, s=0}^{\infty} \lambda^{1+h} \frac{\Gamma(\theta(1+h))(-1)^{h+s}}{\Gamma(-s+(h+1) \theta) \Gamma(1+\mathrm{s})} \\
\times \frac{\exp \left[-(s+1)\left(\frac{\exp \left[-z^{-\beta} \alpha^{\beta}\right]}{1-\exp \left[-z^{-\beta} \alpha^{\beta}\right]}\right)^{2}\right]}{\left\{1-\exp \left[-z^{-\beta_{\alpha} \beta}\right\}^{3}\right.} .
\end{gathered}
$$

Applying the series $\exp (\boldsymbol{\varphi})$ to the term

Equation (9) becomes

$$
\exp \left[-(1+s)\left(\frac{\exp \left[-z^{-\beta} \alpha^{\beta}\right]}{1-\exp \left[-z^{-\beta} \alpha^{\beta}\right]}\right)^{2}\right]
$$

$$
\begin{aligned}
& f_{P B X F r}^{(\lambda, \theta, \alpha, \beta)}(z)=\beta \alpha^{\beta}(1+i)^{j} z^{-(\beta+1)} \exp \left[-x^{-\beta} \alpha^{\beta}\right] \\
& \times \sum_{h, s, l=0}^{\infty} 2 \theta \lambda^{1+h} \frac{\Gamma(\theta(h+1))}{\Gamma(1+s) \mathbf{l}(-1)^{-(s+h+l)} \mathrm{C}_{(\lambda)} \Gamma(\theta(h+1)-s)} \\
& \times \frac{\left\{\exp \left[-z^{-\beta} \alpha^{\beta}\right]\right\}^{2 l+1}}{\left\{1-\exp \left[-z^{-\beta} \alpha^{\beta}\right]\right\}^{2 l+3}} .
\end{aligned}
$$

Consider

$$
\left.(1-\boldsymbol{\varphi})^{-V}\right|_{(|\boldsymbol{\varphi}|<1 \text { and } V>0)}=\sum_{d=0}^{\infty} \frac{\Gamma(V+d)}{d ! \Gamma(V)} \boldsymbol{\varphi}^{d} .
$$

Applying (11) to (10) for the term $\left\{-\exp \left[-z^{-\beta} \alpha^{\beta}\right]+1\right\}^{2 l+3}$, Equation (10) becomes

$$
f_{P B X F r}^{(\lambda, \theta, \alpha, \beta)}(x)=\sum_{l, b=0}^{\infty} v_{j, b} \mathbf{h}_{[b+2+2 l]}(z ; \alpha, \beta),
$$

where

$$
v_{l, k}=2 \theta \lambda^{1+h}(-1)^{l} \frac{\Gamma(3+2 l+b)}{l ! k ! \mathrm{C}_{(\lambda)} \Gamma(2 l+3)[b+2+2 l]}
$$




$$
\sum_{h, s=0}^{\infty} \frac{(-1)^{h+i} \boldsymbol{\Gamma}(\theta h+\theta)}{\boldsymbol{\Gamma}(1+s) \boldsymbol{\Gamma}(-s+\theta h+\theta)(1+s)^{-l}}
$$

and the function $h_{[b+2+2 j]}(z, \beta)$ is the Fr P-D-F with $[b+2+2 j]^{\beta^{-1}} \alpha$ as scale parameter. The C-D-F of the P-BX-Fr can also be re-expressed as a mixture of Fr C-D-Fs given by

$$
F_{P B X F r}^{(\lambda, \theta, \alpha, \beta)}(z)=\sum_{l, b=0}^{\infty} v_{j, k} \mathbf{H}_{[b+2+2 l]}(z ; \alpha, \beta),
$$

where function $\mathbf{H}_{[2(1+j)+b]}(x ; \alpha, \beta)$ is the Fr C-D-F.

\subsection{Quantile and random number generation}

The Q-F of a $\mathrm{R}-\mathrm{V} Z$, where $Z \sim \mathrm{P}-\mathrm{BX}-\operatorname{Fr}(\lambda, \theta, \alpha, \beta)$, is obtained by inverting (5) as

$$
Q(u)=\alpha\left\{-\ln \left[\left(1+\left\{-\ln \left[1-\left(\frac{-\ln \left\{1-u \mathrm{C}_{(\lambda)}\right\}}{\lambda}\right)^{\frac{1}{\theta}}\right]\right\}^{\frac{1}{2}}\right)\right]\right\}^{-\frac{1}{\beta}}
$$

\subsection{Moments}

The $\mathbf{E}\left(Z^{\mathbf{r}}\right)$ or $\mu_{\mathbf{r}}^{\prime}$, comes from (12) as

$$
\mathbf{E}\left(Z^{\mathbf{r}}\right)=\sum_{j, k=0}^{\infty} v_{j, k} \alpha^{\mathbf{r}}[k+2+2 j]^{\mathbf{r} \beta^{-1}} \mathbf{\Gamma}\left(1-\mathbf{r} \beta^{-1}\right)=\left.\mu_{\mathbf{r}}^{\prime}\right|_{(\mathbf{r}<\beta)},
$$

The mean of $Z(\mathbf{E}(Z))$ will be

where

$$
\mathbf{E}(Z)=\sum_{j, k=0}^{\infty} v_{j, k} \alpha[k+2+2 j]^{\beta^{-1}} \boldsymbol{\Gamma}\left(1-\beta^{-1}\right)=\left.\mu_{\mathbf{1}}^{\prime}\right|_{(\mathbf{1}<\beta)},
$$

and

$$
\left.\boldsymbol{\Gamma}(\boldsymbol{\varphi}+1)\right|_{\left(\boldsymbol{\varphi} \in R^{+}\right)}=\prod_{w=0}^{\boldsymbol{\varphi}-1}(\boldsymbol{\varphi}-w),
$$

$$
\int_{0}^{\infty} z^{\varphi-1} \exp (-z) d z=\Gamma(\boldsymbol{\varphi})
$$

Skewness of the P-BX-Fr can range in $(-0.067,1.339)$, whereas the kurtosis of the P-BXFr varies in $(2.916,6.004)$ also the $\mathbf{E}(Z)$ increases as $\lambda$ increases (see Table 1$)$.

\subsection{Incomplete moments (IM)}

The $\mathbf{r}$ th IM of $Z$ is defined as $\mathbf{m}_{\mathbf{r}}(y)=\int_{-\infty}^{y} \quad z^{\mathbf{r}} f(z) d z$. We can write from (12)

$$
\left.\mathbf{m}_{\mathbf{r}}(y)\right|_{(\mathbf{r}<\beta)}=\sum_{l, k=0}^{\infty} v_{l, k} \alpha^{\mathbf{r}}[k+2+2 j]^{\mathbf{r} \beta^{-1}} \Gamma\left(1-\mathbf{r} \beta^{-1},\left(\frac{\alpha}{y}\right)^{\beta}\right) .
$$

Setting $\mathbf{r}=1$ in (15) gives the 1st incomplete moment of $X$ as

$$
\left.\mathbf{m}_{1}(y)\right|_{(1<\beta)}=\sum_{l, k=0}^{\infty} v_{l, k} \alpha[k+2+2 l]^{\beta^{-1}} \Gamma\left(1-\beta^{-1},\left(\frac{\alpha}{y}\right)^{\beta}\right),
$$

where $\gamma(\boldsymbol{\varphi}, q)$ is the incomplete gamma function, where 


$$
\begin{aligned}
& \left.\gamma(\boldsymbol{\varphi}, q)\right|_{(\boldsymbol{\varphi} \neq 0,-1,-2, \ldots)}=\int_{0}^{q} z^{\boldsymbol{\varphi}-1} \exp (-z) d z \\
& =q^{\boldsymbol{\varphi}}\left\{1 \mathrm{~F}_{1}[\boldsymbol{\varphi} ; \boldsymbol{\varphi} a+1 ;-q]\right\} \frac{1}{\boldsymbol{\varphi}} \\
& =\sum_{\mathbf{k}=0}^{\infty} q^{\boldsymbol{\varphi}+\mathbf{k}} \frac{(-1)^{\mathbf{k}}}{k !(\varphi+\mathbf{k})},
\end{aligned}
$$

the function $1 \mathrm{~F}_{1}[\because, \cdot$,$] is a called the confluent hypergeometric function,$

and

$$
\left.\boldsymbol{\Gamma}(\boldsymbol{\varphi}, q)\right|_{(x>0)}=\int_{q}^{\infty} z^{\boldsymbol{\varphi}-1} \exp (-z) d z,
$$

$$
\boldsymbol{\Gamma}(\boldsymbol{\varphi}, q)+\gamma(\boldsymbol{\varphi}, q)=\boldsymbol{\Gamma}(\boldsymbol{\varphi})
$$

\subsection{Generating function (M-G-F)}

The M-G-F, $\boldsymbol{M}(t)=\mathbf{E}(\exp (t Z))$, is derived from (12) as

$$
\left.\boldsymbol{M}(t)\right|_{(r<\beta)}=\sum_{l, k, \mathbf{r}=0}^{\infty} v_{l, k}\left(\frac{t^{\mathbf{r}}}{\mathbf{r} !}\right) \alpha^{\mathbf{r}}[k+2+2 l]^{\mathbf{r} \beta^{-1}} \boldsymbol{\Gamma}\left(1-\mathbf{r} \beta^{-1}\right),
$$

Let the generalized Wright hypergeometric function $\left(p \mathbf{W}_{q}\right)$ defined as

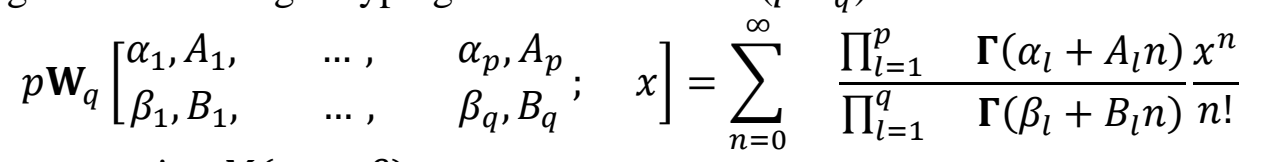

Then, we can write $M(t ; \alpha, \beta)$ as

$$
\boldsymbol{M}(t ; \alpha, \beta)={ }_{1} \mathbf{W}_{0}\left[\begin{array}{c}
\left(1,-\beta^{-1}\right) \\
-
\end{array} ; t\right] .
$$

Combining (12) and (16), we obtain the M-G-F of $Z$, say $\boldsymbol{M}(t)$, as

$$
\boldsymbol{M}(t)=\sum_{l, k=0}^{\infty} v_{l, k} \quad{ }_{1} \mathbf{W}_{0}\left[\begin{array}{c}
\left(1,-\beta^{-1}\right) \\
-
\end{array} \alpha[k+2+2 l]^{\beta^{-1}} t\right] .
$$

\section{Estimation}

Consider any random sample (R-S) of size $m$ from the P-BX-Fr, then the log likelihood $(\log L)$ function can be expressed as

$$
\begin{gathered}
\log L=m+m \log \theta+m_{m} \log \lambda+m \log \beta+m \beta \log \alpha \\
-m \log \mathrm{C}_{(\lambda)}-(\beta+1) \sum_{s=1}^{m} \log z_{s}-3 \log \left(-\xi_{s}+1\right) \\
+2 \sum_{s=1}^{n} \log \xi_{s}-\lambda \sum_{s=1}^{m}\left[-\exp \left(-\tau_{s}\right)+1\right]^{\theta}-\sum_{s=1}^{m} \tau_{s} \\
+(\theta-1) \sum_{s=1}^{m} \log \left[-\exp \left(-\tau_{s}\right)+1\right]
\end{gathered}
$$

where

$$
\xi_{s}=\exp \left[-\left(\alpha z_{s}{ }^{-1}\right)^{\beta}\right]
$$




$$
\tau_{s}=\left(\frac{\xi_{s}}{-\xi_{s}+1}\right)^{2}
$$

The maximum likelihood method and its procedures are available with details.

\section{Real data modeling}

Consider the following competitive models

\begin{tabular}{|l|l|}
\hline Competitive models & Author \\
\hline Kumaraswamy-Fr (Kum Fr) & (Mead, and Abd - Eltawab, (2014)) \\
\hline exponentiated-Fréchet (E Fr) & (Nadarajah, and Kotz, (2003)) \\
\hline Weibull-Fr & (Afify, et al. (2016-b)) \\
\hline Mershall-Olkin-Fr (MO Fr) & (Krishna, et al., (2013)) \\
\hline Beta-Fr (BFr) & (Barreto-Souza, et al., (2011)) \\
\hline Gamma-extended-Fr (G E Fr) & (Silva, et. al., (2013)) \\
\hline Transmuted-Fr (T Fr) & (Mahmoud and Mandouh, (2013)) \\
\hline
\end{tabular}

\begin{tabular}{|l|l|l|l|}
\hline Data & $\mathrm{n}$ & Called & Author \\
\hline $1 s t$ & 100 & Stress data & Nichols \& Padgett (2006) \\
\hline $2 n d$ & 63 & Glass fibers data & Smith \& Naylor (1987) \\
\hline
\end{tabular}

For comparing models, we will consider: AI_C (Akaike Information Criterion), CAI_C (Consistent AIC), BI_C (Bayesian IC), HQI_C (Hannan - Quinn-IC) and the maximized $\log L$. The total-time-test (T-T-T) plots the two real data sets are given in Figure 3. From Figure 3, we conclude that the empirical H-R-Fs (E-H-R-Fs) of the two data can be increasing. In Tables 2 and 3, we compared the P-BX-Fr with other Fr versions. The P$\mathrm{BX}-\mathrm{Fr}$ version gives the lowest statistics among all fitted versions. So, the new one may be chosen as the best one. Figure 4 display plots E-P-D-F, P-P plot, E-C-D-F, estimated H-R$\mathrm{F}$ and Kaplan-Meier survival plot (K-M-S) of the P-BX-Fr model for the 1 st data. Figure 5 display the plots of E-P-D-F, P-P plot, E-C-D-F, E-H-R-F and K-M-S plot of the proposed P-BX-Fr model for the 2 nd data.

\section{Conclusions}

In this paper, a new wider extension of the Fr model is introduced. The new Fr version was based on the well-known Z-T-P model. The P-D-F of the new Fr model can be right skewed and unimodal also it can be left skewed. The H-R-F of the new Fr model can be bathtub (U-shape), unimodal-bathtub (unimodal-U), increasing and decreasing. Some of its properties are mathematically derived and numerically studied. The method of ML is used to estimate the unknown parameters.

\section{Acknowledgement}

This research was funded by the Deanship of Scientific Research at Princess Nourah bint Abdulrahman University through the Fast-track Research Funding Program. 


\section{References}

1. Aarset, M. V. (1987). How to identify a bathtub hazard rate. IEEE Transactions on Reliability, 36(1), 106-108

2. Afify, A. Z., Yousof, H. M. Cordeiro, G.M. and Ahmad, M. (2016a). The Kumaraswamy Marshall-Olkin Fréchet distribution: Properties and Applications, Journal of ISOSS, 2(1), 41-58.

3. Afify, A. Z., Yousof, H. M., Cordeiro, G. M., Ortega, E. M. M. and Nofal, Z. M. (2016b). The Weibull Fréchet distribution and its applications. Journal of Applied Statistics, 43(14), 2608--2626.

4. Alizadeh, M., Yousof, H. M., Afify, A. Z., Cordeiro, G. M., \& Mansoor, M. (2018). The complementary generalized transmuted Poisson-G family of distributions. Austrian Journal of Statistics, 47(4), 60-80.

5. Alizadeh, M., Yousof, H. M., Rasekhi, M., \& Altun, E. (2018). The odd loglogistic Poisson-G Family of distributions. Journal of Mathematical Extensions.

6. Barreto-Souza, W. M., Cordeiro, G. M. and Simas, A. B. (2011). Some results for beta Fréchet distribution. Communications in Statistics - Theory and Methods, 40, 798-811.

7. Brito, E., Cordeiro, G. M., Yousof, H. M., Alizadeh, M. and Silva, G. O. (2017). Topp-Leone Odd Log-Logistic Family of Distributions, Journal of Statistical Computation and Simulation, 87(15), 3040-3058.

8. Chakraborty, S., Handique, L., Altun, E. and Yousof, H. M. (2018). A new statistical model for extreme values: mathematical properties and applications. International Journal of Open Problems in Computer Science and Mathematics, 12(1), 1-18.

9. Cordeiro, G. M., Yousof, H. M., Ramires, T. G. and Ortega, E. M. M. (2018). The Burr XII system of densities: properties, regression model and applications. Journal of Statistical Computation and Simulation, 88(3), 432-456.

10. Hamedani G. G., Altun, E, Korkmaz, M. C., Yousof, H. M. and Butt, N. S. (2018). A new extended $\mathrm{G}$ family of continuous distributions with mathematical properties, characterizations and regression modeling. Pak. J. Stat. Oper. Res., 14 (3), 737-758.

11. Hamedani G. G. Rasekhi, M., Najibi, S. M., Yousof, H. M. and Alizadeh, M., (2019). Type II general exponential class of distributions. Pak. J. Stat. Oper. Res., forthcoming.

12. Hamedani G. G. Yousof, H. M., Rasekhi, M., Alizadeh, M., Najibi, S. M. (2017). Type I general exponential class of distributions. Pak. J. Stat. Oper. Res., XIV(1), 39-55.

13. Korkmaz, M. C., Alizadeh, M., Yousof, H. M. and Butt, N. S. (2018). The generalized odd Weibull generated family of distributions: statistical properties and applications. Pak. J. Stat. Oper. Res., 14 (3), 541-556.

14. Korkmaz, M. C., Altun, E., Yousof, H. M. and Hamedani G. G. (2019). The Odd Power Lindley Generator of Probability Distributions: Properties, Characterizations and Regression Modeling, International Journal of Statistics and Probability, 8(2). 70-89.

15. Korkmaz, M. C. Yousof, H. M. and Ali, M. M. (2017). Some Theoretical and Computational Aspects of the Odd Lindley Fréchet Distribution, Journal of Statisticians: Statistics and Actuarial Sciences, 2, 129-140

16. Krishna, E., Jose, K. K., Alice, T. and Ristic, M. M. (2013). The Marshall-Olkin 
Fréchet Distribution. Communications in Statistics - Theory and Methods, 42, 4091-4107.

17. Mahmoud, M. R. and Mandouh, R. M. (2013). On the Transmuted Fréchet Distribution. Journal of Applied Sciences Research, 9, 5553-5561.

18. Mead, M. E. and Abd-Eltawab A. R. (2014). A note on Kumaraswamy-Fréchet Distribution. Aust. J. Basic and Appl. Sci., 8, 294-300.

19. Mubarak, M. (2011). Parameter estimation based on the Fréchet Progressive Type II censored data with binomial removals. Journal of Quality, Statistics and Reliability 2012 (2011).

20. Nadarajah, S. and Gupta, A. K. (2004). The Beta Fréchet Distribution. Far East Journal of Theoretical Statistics, 14, 15-24.

21. Kotz, S. and Nadarajah, S. (2000). Extreme Value Distributions: Theory and Applications. Imperial College Press, London.

22. Nadarajah, S. and Kotz, S. (2003). The exponentiated Fréchet distribution. Interstat Electronic Journal, 1-7.

23. Nichols, M. D, Padgett, W. J. (2006). A Bootstrap control chart for Weibull percentiles. Quality and Reliability Engineering International, 22, 141-151.

24. Silva, R. V. D., de Andrade, T. A., Maciel, D., Campos, R. P., and Cordeiro, G. M. (2013). A new lifetime model: The gamma extended Fréchet distribution. Journal of Statistical Theory and Applications, 12, 39-54.

25. Smith, R. L. and Naylor, J. C. (1987). A comparison of maximum likelihood and Bayesian estimators for the three-parameter Weibull distribution. Applied Statistics, 36, 358-369.

26. Yousof, H., Afify, A. Z., Alizadeh, M., Hamedani, G. G., Jahanshahi, S., \& Ghosh, I. (2018). The Generalized Transmuted Poisson-G Family of Distributions: Theory, Characterizations and Applications. Pakistan Journal of Statistics and Operation Research, 14(4), 759-779.

27. Yousof, H. M., Afify, A. Z., Alizadeh, M., Butt, N. S., Hamedani, G. G. and Ali, M. M. (2015). The transmuted exponentiated generalized-G family of distributions. Pak. J. Stat. Oper. Res., 11, 441-464.

28. Yousof, H. M., Afify, A. Z., Ebraheim, A. N., Hamedani, G. G. and Butt, N. S. (2016). On six-parameter Fréchet distribution: properties and applications, Pak. J. Stat. Oper. Res., 12, 281-299.

29. Yousof, H. M., Alizadeh, M., Jahanshahiand, S. M. A., Ramires, T. G., Ghosh, I. and Hamedani G. G. (2017). The transmuted Topp-Leone G family of distributions: theory, characterizations and applications, Journal of Data Science. 15, 723-740.

30. Yousof, H. M., Altun, E. and Hamedani, G. G. (2018a). A new extension of Frechet distribution with regression models, residual analysis and characterizations. Journal of Data Science, 16(4), 743-770.

31. Yousof, H. M., Jahanshahi, S. M., Ramires, T. G Aryal, G. R. and Hamedani G. G. (2018b). A new distribution for extreme values: regression model, characterizations and applications. Journal of Data Science, 16(4), 677-706.

\section{Appendix}



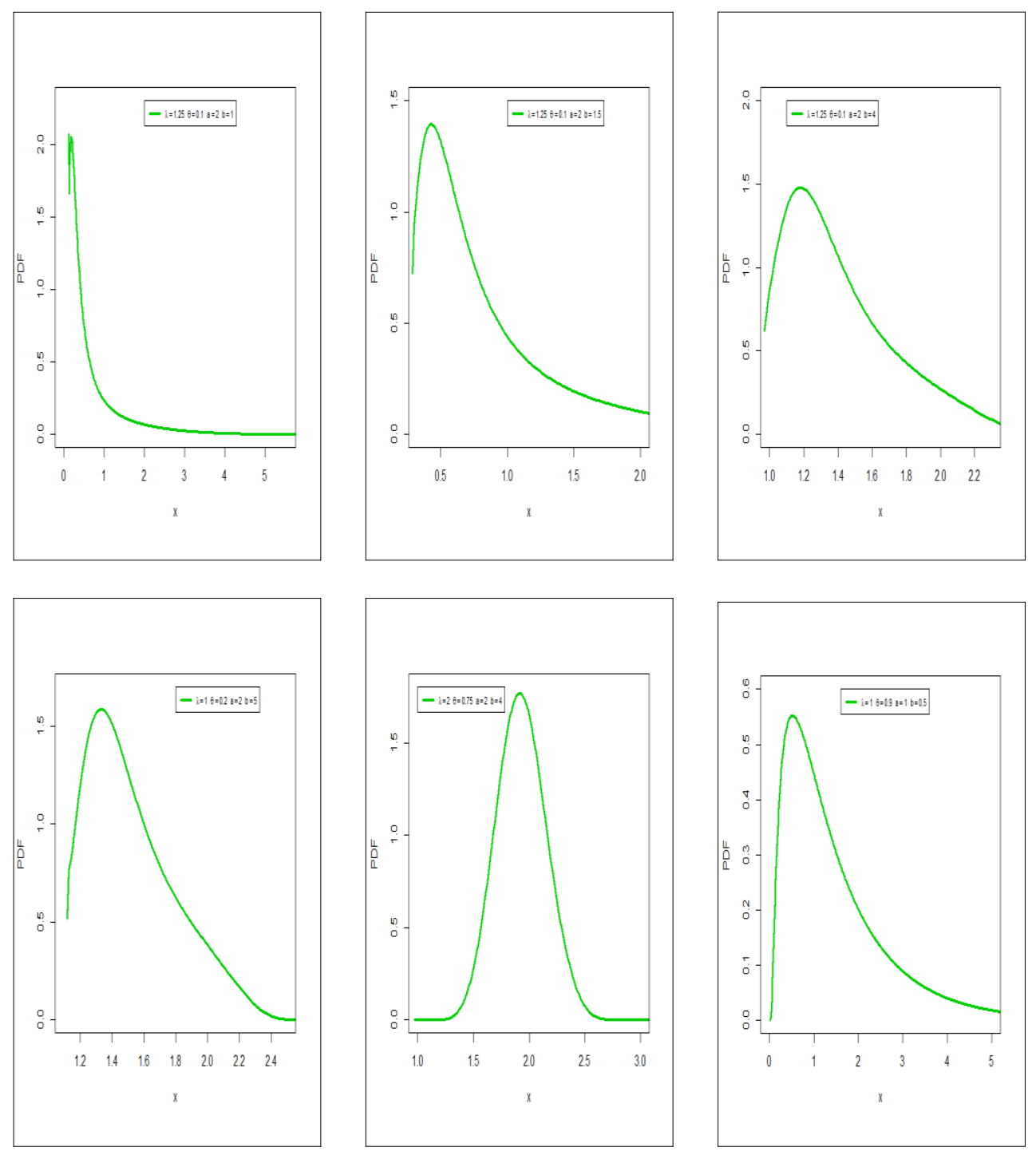

Figure 1:P.D.F.s of the new version. 
Mohamed G. Khalil \& Rezk H.
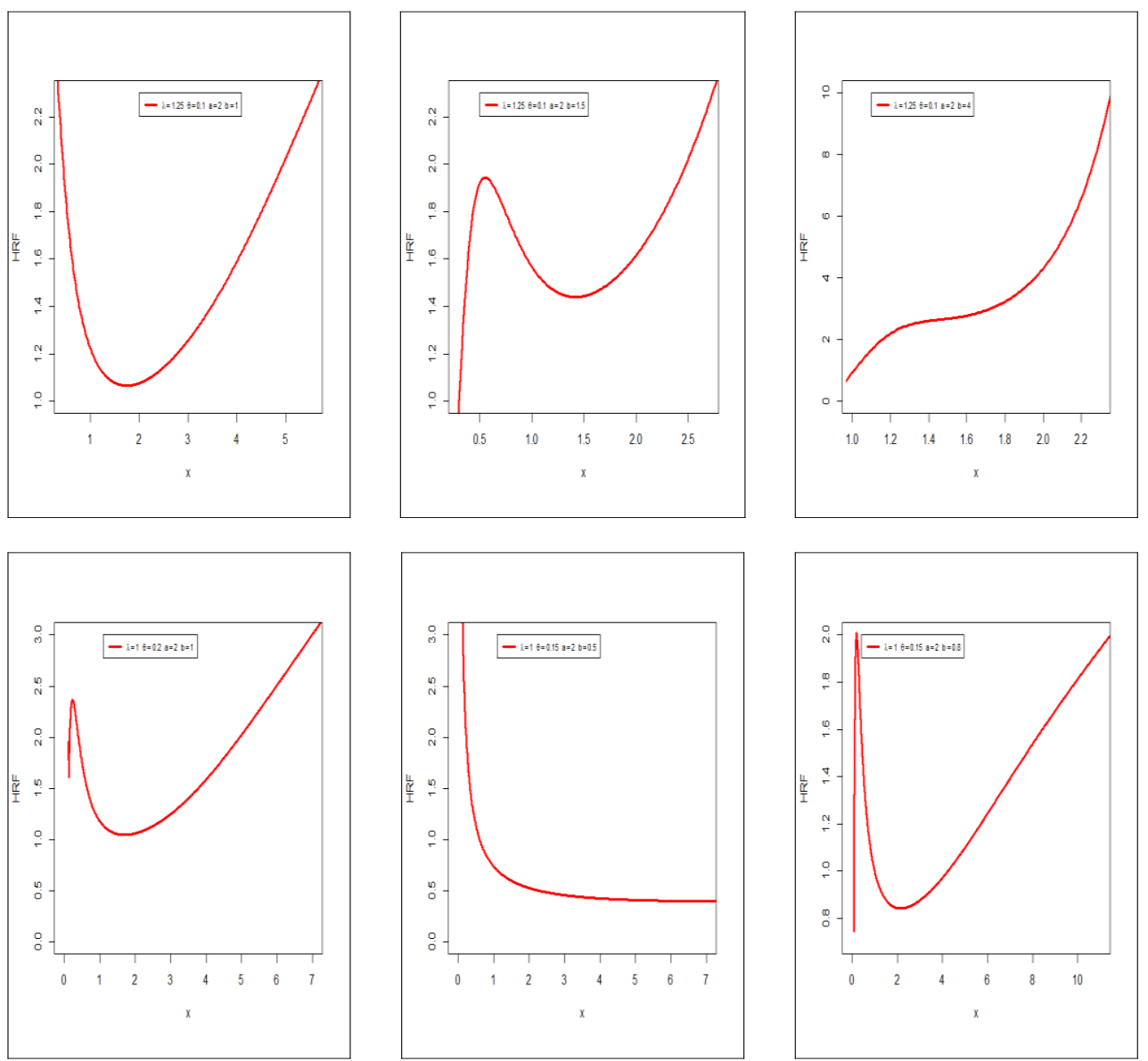

Figure 2: H.R.F.s of the new version. 
Table 1: measures of the P-BX-Fr

version with $\alpha=1.25, \beta=0.75$, different values of $\lambda$ and $\theta$.

\begin{tabular}{|c|c|c|c|c|c|}
\hline$\lambda$ & $\theta$ & Mean & Variance & Skewness & Kurtosis \\
\hline 50 & 10 & 2.081183 & 0.0437062 & -0.268461 & 3.125746 \\
\hline 20 & & 2.270348 & 0.0684343 & -0.067069 & 3.115156 \\
\hline 10 & & 2.455186 & 0.1095668 & 0.2804898 & 3.777957 \\
\hline 5 & & 2.705083 & 0.2181157 & 0.9647563 & 5.976804 \\
\hline 3 & & 2.918478 & 0.3435836 & 1.079125 & 5.600215 \\
\hline 1 & & 3.252673 & 0.5195756 & 0.8665094 & 4.342047 \\
\hline-1 & & 3.672990 & 0.6303753 & 0.5660974 & 3.632238 \\
\hline-3 & & 4.059918 & 0.6113683 & 0.4306407 & 3.640462 \\
\hline-5 & & 4.345674 & 0.5467593 & 0.4745266 & 3.824413 \\
\hline-10 & & 4.750441 & 0.4510728 & 0.6734854 & 4.035540 \\
\hline-20 & & 5.121649 & 0.3945953 & 0.7893729 & 4.202697 \\
\hline-50 & & 5.57532 & 0.3485676 & 0.8619313 & 4.362489 \\
\hline & & & & & \\
\hline 50 & 1.5 & 0.656871 & 0.0276661 & 0.2000253 & 2.916146 \\
\hline 20 & & 0.826048 & 0.0563583 & 0.3857478 & 3.196063 \\
\hline 10 & & 1.009971 & 0.1104200 & 0.7382564 & 4.535035 \\
\hline 5 & & 1.280310 & 0.2634135 & 1.3386010 & 7.028746 \\
\hline 3 & & 1.521799 & 0.4446594 & 1.3046860 & 6.003620 \\
\hline 1 & & 1.908314 & 0.7034265 & 0.9623336 & 4.29767 \\
\hline-1 & & 2.401288 & 0.8719591 & 0.5815450 & 3.429711 \\
\hline-3 & & 2.858543 & 0.8488771 & 0.3894757 & 3.398211 \\
\hline-5 & & 3.197011 & 0.7534608 & 0.3951807 & 3.582307 \\
\hline-10 & & 3.674373 & 0.6044304 & 0.5725431 & 3.790513 \\
\hline-20 & & 4.106018 & 0.5118386 & 0.6976685 & 3.958058 \\
\hline-50 & & 4.623793 & 0.4359177 & 0.7865258 & 4.142977 \\
\hline
\end{tabular}




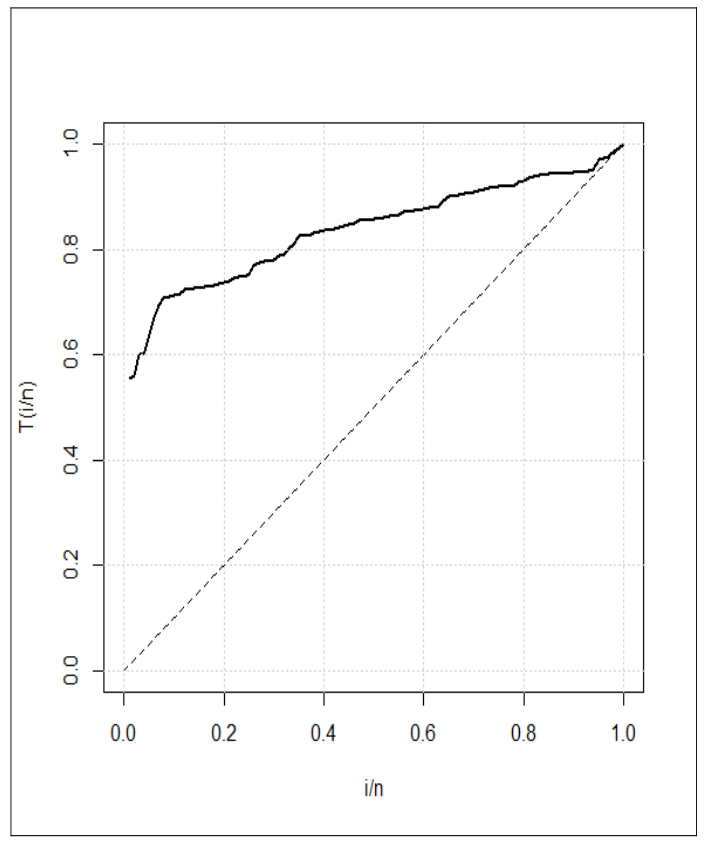

Data set I

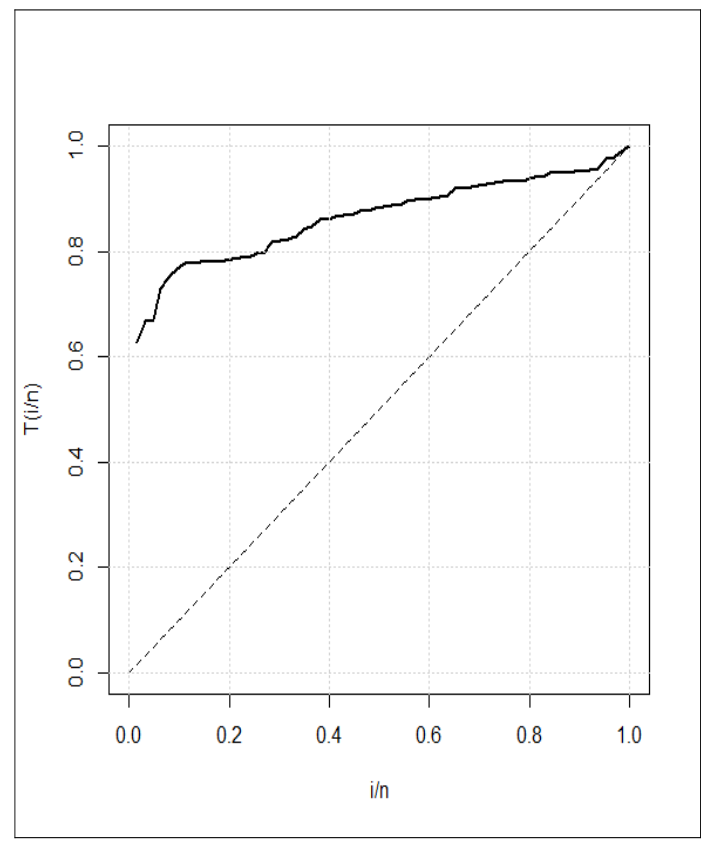

Data set II

Figure 3: gives the TTT plots.

Table 2

\begin{tabular}{|c|c|}
\hline Model & Goodness of fit criteria \\
\hline & BI_C, AI_C, CAI_C, HQI_C, $-2 \ell$ \\
\hline P-BX-Fr & 133.06, 123.06, 290.19, 126.86, 114.60 \\
\hline W_Fr & $304.93,294.58,294.94,298.67,286.52$ \\
\hline E_Fr & $303.55,295.66,296.01,298.88,289.74$ \\
\hline Kum_Fr & $307.56,297.10,297.52,301.34,289.13$ \\
\hline B_Fr & $321.60,311.10,311.58,315.42,303.19$ \\
\hline GE_Fr & $332.43,312.00,312.42,316.20,304.00$ \\
\hline Fr & $353.52,348.30,348.43,350.38,344.36$ \\
\hline T_Fr & $358.32,350.46,350.72,353.62,344.58$ \\
\hline MO_Fr & $359.10,351.29,351.64,354.54,345.34$ \\
\hline
\end{tabular}


Table 3

\begin{tabular}{|c|c|c|c|c|}
\hline Model & \multicolumn{4}{|c|}{ Estimates } \\
\hline $\operatorname{P-BX}-\operatorname{Fr}(\lambda, \theta, \alpha, \beta)$ & 4.9 & 3.452 & 1.0310 & 0.742 \\
\hline & (1.247) & (1.024) & (0.193) & 0.117 \\
\hline \multirow{2}{*}{ Kum_Fr $(\alpha, \beta, a, b)$} & 2.0556 & 0.4654 & 6.2815 & 224.18 \\
\hline & $(0.071)$ & $(0.00701)$ & (0.063) & (0.164) \\
\hline \multirow[t]{2}{*}{ W_Fr $(\alpha, \beta, a, b)$} & 2.2231 & 0.355 & 6.9721 & 4.9179 \\
\hline & (11.409) & $(0.411)$ & (113.811) & (3.756) \\
\hline \multirow[t]{2}{*}{ GE_Fr( $\alpha, \beta, a, b)$} & 1.3692 & 0.4776 & 27.6452 & 17.4581 \\
\hline & (2.017) & $(0.133)$ & $(14.136)$ & (14.818) \\
\hline \multirow{2}{*}{$\mathrm{B} \operatorname{Fr}(\alpha, \beta, \mathrm{a}, \mathrm{b})$} & 1.6097 & 0.4046 & 22.0143 & 29.7617 \\
\hline & $(2.498)$ & $(0.108)$ & $(21.432)$ & $(17.479)$ \\
\hline \multirow[t]{2}{*}{ T_Fr $(\alpha, \beta, a)$} & 1.9315 & 1.7435 & 0.0819 & \\
\hline & $(0.097)$ & $(0.076)$ & (0.198) & \\
\hline \multirow[t]{2}{*}{ E_Fr( $(\alpha, \beta, a)$} & 69.1489 & 0.5019 & 145.3275 & \\
\hline & $(57.349)$ & $(0.08)$ & (122.924) & \\
\hline \multirow[t]{2}{*}{ MO $\operatorname{Fr}(\alpha, \beta, a)$} & 2.3066 & 1.5796 & 0.5988 & \\
\hline & $(0.498)$ & $(0.16)$ & $(0.3091)$ & \\
\hline \multirow[t]{2}{*}{$\operatorname{Fr}(\alpha, \beta)$} & 1.8705 & 1.7766 & & \\
\hline & $(0.112)$ & $(0.113)$ & & \\
\hline
\end{tabular}

Table 4

\begin{tabular}{|c|c|}
\hline Model & Goodness of fit criteria \\
\hline & BI_C, AI_C, CAI_C, HQI_C, $-2 \ell$ \\
\hline P-BX-Fr & $\mathbf{5 8 . 1 5 , 4 9 . 5 8 , 5 0 . 0 3 , 5 2 . 9 0 , 4 1 . 4 0}$ \\
\hline B_Fr & $77.20,68.62,69.34,72.03,60.65$ \\
\hline GE_Fr & $78.10,69.60,70.32,72.90,61.63$ \\
\hline Fr & $102.00,97.720,97.920,99.440,93.79$ \\
\hline T_Fr & $106.50,100.14,100.51,102.63,94.19$ \\
\hline MO_Fr & $108.20,101.74,102.16,104.26,95.75$ \\
\hline
\end{tabular}

Table 5

\begin{tabular}{|c|c|c|c|c|}
\hline Model & \multicolumn{4}{|c|}{ Estimates } \\
\hline \multirow{2}{*}{$\operatorname{P-BX}-\operatorname{Fr}(\lambda, \theta, \alpha, \beta)$} & 4.492 & 19.999 & 0.383 & 0.506 \\
\hline & (1.778) & $(9.241)$ & (0.184) & $(\mathbf{0 . 1 0 9 )}$ \\
\hline \multirow[t]{2}{*}{ GE_Fr $(\alpha, \beta, a, b)$} & 1.6625 & 0.7421 & 32.112 & 13.2688 \\
\hline & $(0.952)$ & (0.197) & (17.397) & $(9.967)$ \\
\hline \multirow[t]{2}{*}{ B_Fr $(\alpha, \beta, a, b)$} & 2.0518 & 0.6466 & 15.0756 & 36.9397 \\
\hline & $(0.986)$ & $(0.163)$ & (12.057) & (22.649) \\
\hline \multirow[t]{2}{*}{ MO_Fr $(\alpha, \beta, a)$} & 1.5441 & 2.3876 & 0.4816 & \\
\hline & $(0.226)$ & $(0.253)$ & $(0.252)$ & \\
\hline \multirow{2}{*}{ T_Fr( $(\alpha, \beta, a)$} & 1.3068 & 2.7898 & 0.1298 & \\
\hline & $(0.034)$ & $(0.165)$ & $(0.208)$ & \\
\hline \multirow[t]{2}{*}{$\operatorname{Fr}(\alpha, \beta)$} & 1.264 & 2.888 & & \\
\hline & $(0.059)$ & $(0.234)$ & & \\
\hline
\end{tabular}


Mohamed G. Khalil \& Rezk H.
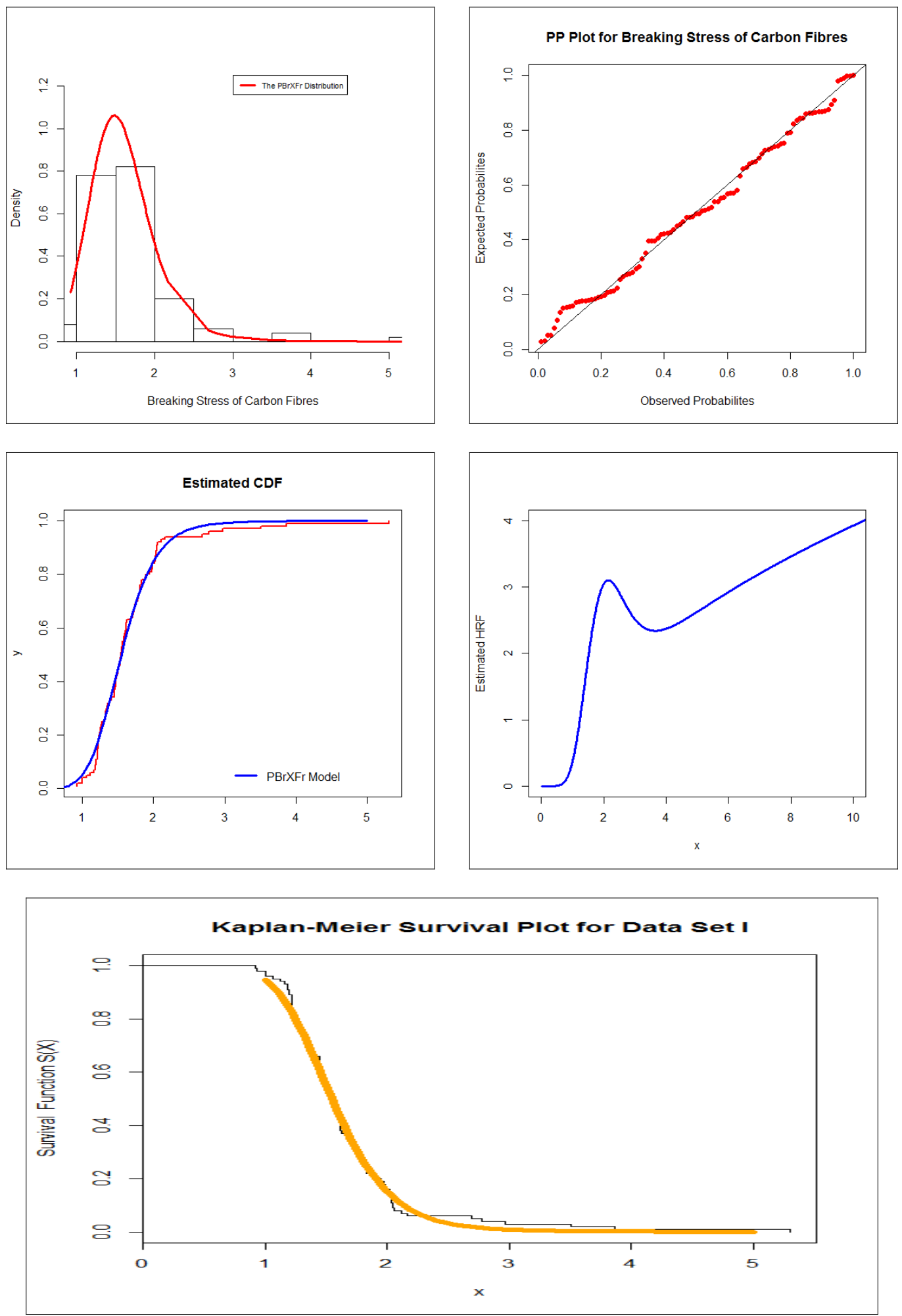

Figure 4. 

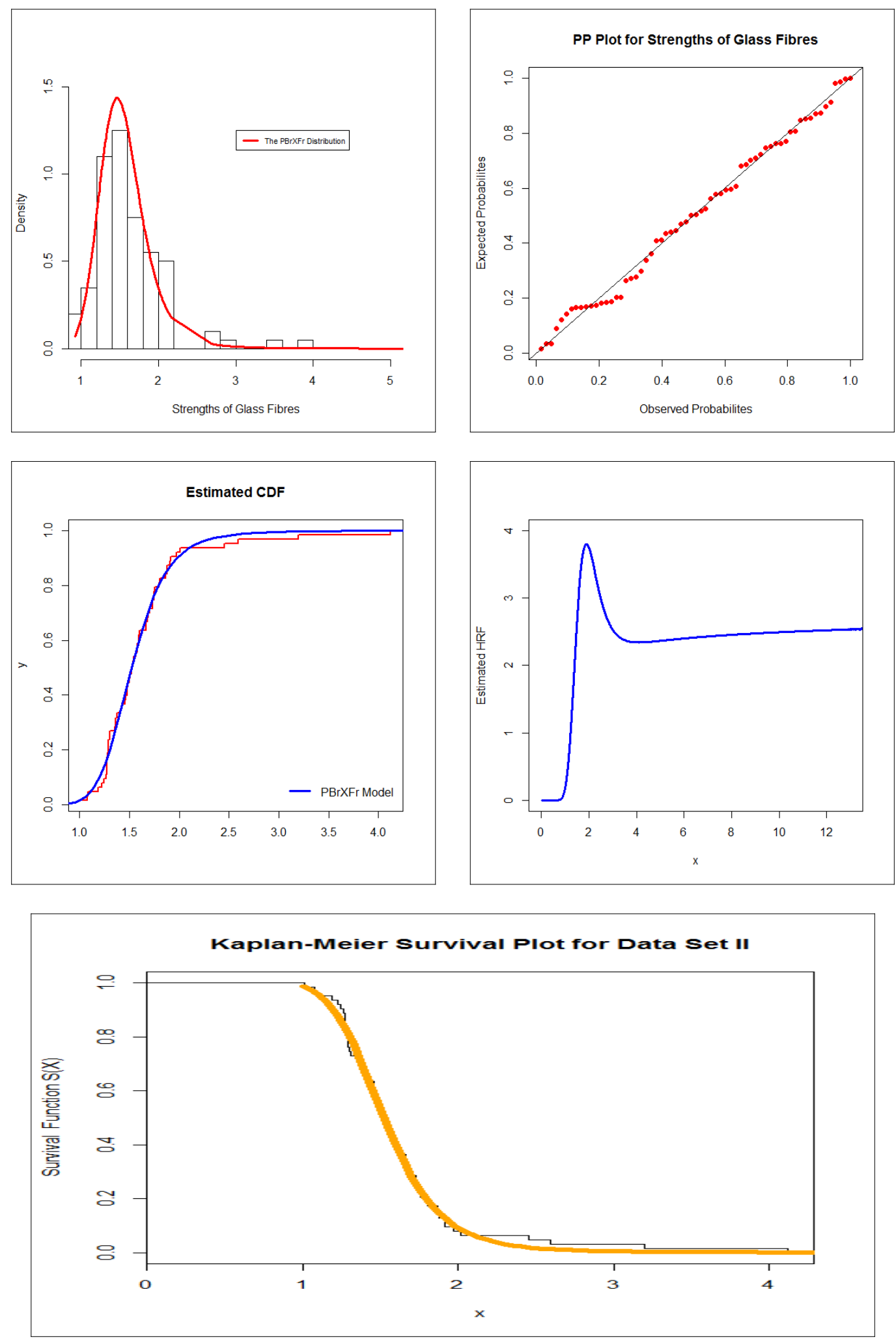

Figure 5. 